Research Article

\title{
Trends in Stroke Prevention between 2014 and 2018 in Hospitalized Atrial Fibrillation Patients
}

\author{
B. Bielecka $\mathbb{D}^{1},{ }^{1}$ I. Gorczyca $\mathbb{D}^{1,2}$ O. Jelonek $\mathbb{D},{ }^{1,2}$ and B. Wożakowska-Kapłon $\mathbb{D}^{1,2}$ \\ ${ }^{1} 1$ st Clinic of Cardiology and Electrotherapy, Swietokrzyskie Cardiology Center, Kielce 25-736, Poland \\ ${ }^{2}$ Collegium Medicum, The Jan Kochanowski University, Kielce 25-369, Poland \\ Correspondence should be addressed to I. Gorczyca; iwona.gorczyca@interia.pl
}

Received 19 November 2020; Revised 8 January 2021; Accepted 28 January 2021; Published 9 February 2021

Academic Editor: Paolo Severino

Copyright (c) 2021 B. Bielecka et al. This is an open access article distributed under the Creative Commons Attribution License, which permits unrestricted use, distribution, and reproduction in any medium, provided the original work is properly cited.

In recent years, significant changes in stroke prophylaxis in patients with atrial fibrillation (AF) have been observed. Nonvitamin K antagonist oral anticoagulants (NOACs) are more commonly used in the prevention of thromboembolic complications in patients with AF. The aim of the study was to evaluate recommended stroke prophylaxis in patients with AF and to identify predictors of using NOACs in patients treated with anticoagulant therapy. The present study was a retrospective, observational, single-center study which included consecutively hospitalized patients in the reference cardiology center from January 2014 to December 2018. In the study group of 4027 patients with AF, to prevent thromboembolic complications, OACs were used in 3680 patients (91.4\%), an antiplatelet drug(s) was used in 124 patients (3.1\%), and 223 patients (5.5\%) did not undergo any thromboembolic event prevention. In the group of 3680 patients treated with OACs, 2311 patients (62.8\%) received NOACs and 1639 patients (37.2\%), VKAs. Independent predictors of the use of NOACs were age (OR, 1.02; 95\% CI, 1.01-1.03; $P<0.001)$, a previous thromboembolic event $(\mathrm{OR}, 1.29 ; 95 \% \mathrm{CI}, 1.01-1.65 ; P=0.04)$, nonpermanent AF (OR, 1.61; 95\% CI, 1.34-1.93; $P<0.001$ ), and eGFR (OR, 1.22; 95\% CI, 1.02-1.46; $P=0.03$ ). Between 2014 and 2018, an increase of patients treated with OACs, mainly with NOACs, was observed. Age, past thromboembolic complications, nonpermanent AF, and preserved renal function determined the choice of NOACs.

\section{Introduction}

Atrial fibrillation (AF) is the most frequent supraventricular arrhythmia. It affects roughly $1-2 \%$ of the population and is connected with a fivefold increase of a thromboembolic complication risk $[1,2]$. It is estimated that among middle-aged people every fourth person will suffer from AF. The risk of thromboembolic complications in patients with AF is not homogeneous and depends on age, sex, and comorbidities. To estimate the risk of thromboembolic complications in patients with $\mathrm{AF}$, the $\mathrm{CHA}_{2} \mathrm{DS}_{2}$-VASc score is used [3]. The guidelines of the European Society of Cardiology point that anticoagulant therapy is advised in patients with a high risk of thromboembolic complications. Anticoagulant prophylaxis should not be applied in patients of low risk of thromboembolic complications [4]. Vitamin $\mathrm{K}$ antagonists (VKAs) and non-vitamin $\mathrm{K}$ antagonist oral anticoagulants (NOACs) appeared to be effective in the prevention of thromboembolic complications in patients with AF [5-8]. In recent years, it has been possible to observe a change in stroke prophylaxis in $\mathrm{AF}$ patients, which results from more and more common NOAC administration in the prevention of thromboembolic complications $[9,10]$. Although the guidelines clearly define indications for OAC application, it is not easy to implement them in clinical practice. In international registries, both patients of low thromboembolic risk treated with OACs and patients of high thromboembolic risk not receiving OACs are observed [11-13].

The aim of the study was to evaluate recommended anticoagulant prophylaxis in patients with AF, with a particular reference to the assessment of NOAC application frequency and an attempt to identify factors that clinched the use of NOACs in patients treated with OACs. 


\section{Materials and Methods}

2.1. Study Group. The study includes all consecutive patients with AF hospitalized in a referential cardiology center from January 2014 to December 2018, who were hospitalized for urgent and planned reasons. Patients were included if they were at least 18 years of age and had a history of AF documented by electrocardiography or in their medical history.

Mechanical heart valve, moderate or severe mitral valve, and death during hospitalization were the exclusion criteria.

$\mathrm{AF}$ was diagnosed on the basis of the definition of the European Society of Cardiology, according to which arrhythmia can be identified using an electrocardiogram showing irregular atrial rhythm lasting longer than 30 seconds [3].

2.2. Assessed Parameters. Baseline characteristics concerning medical history, AF type, demographics, diagnostic test results, and pharmacotherapy were collected.

CHA2DS2-VASc (congestive heart failure, hypertension, age $\geq 75$ years, diabetes mellitus, stroke/transient ischaemic attack, vascular disease, age 65-74 years, and sex category) score was used to make an assessment of thromboembolic risk whereas bleeding risk was defined with HAS-BLED (hypertension, abnormal renal/liver function, stroke, bleeding, labile INR, elderly (>65 years), and drug/ alcohol consumption) [13].

The estimated glomerular filtration rate (eGFR) used to assess patients' kidney function was calculated using the Modification of Diet in Renal Disease (MDRD) Study equation.

The study was approved by the Ethics Committee of the Swietokrzyskie Medical Chamber in Kielce. Informed consent from the patients was not required by the Ethics Committee.

2.3. Management of Antithrombotic Therapy. Antithrombotic therapy recommended during the patients' discharge from the hospital was evaluated. Three types of regiments were defined: $\mathrm{OAC} \pm$ antiplatelet drug (AP), AP alone, and no antithrombotic treatments. OAC group included VKAs, apixaban, dabigatran, and rivaroxaban alone or with AP. Edoxaban has been registered in Europe as a drug for preventing thromboembolic complications in patients with AF; however, it is not available in Poland. AP group included acetylsalicylic acid (ASA) or/and clopidogrel, ticagrelor, and prasugrel. No antithrombotic treatment was defined by the absence of OAC and AP prescription.

2.4. Statistical Analysis. The normality of data distributions was tested using the Kolmogorov-Smirnov test. The hypothesis of normality was rejected for most of the analysed variables; therefore, nonparametric methods were used. The level of significance was set as $\alpha=0.05$. Descriptive statistics are presented as means and standard deviation or median and interquartile range. The distribution of qualitative data was presented as frequency and percentages. Frequencies were compared using the $\chi^{2}$ test. The distributions of quantitative variables were compared using the Mann-Whitney $U$ test. The odds ratios (OR) together with a $95 \%$ confidence interval (CIs) were determined using a univariate logistic regression model. A multivariate logistic regression analysis was used to explore the variables associated with increasing the chances of using non-vitamin $\mathrm{K}$ antagonist oral anticoagulants (NOACs); the variables which presented statistically significant OR were included. Statistical analyses were performed with SPSS version 25 (IBM).

\section{Results and Discussion}

3.1. Characteristics of the Study Group. In the study group of 4027 patients with AF, the mean age was 71.7 (11.4) years; $42.6 \%$ were women. Most often comorbidities were hypertension-3117 patients (77.4\%), heart failure-2520 patients (62.6\%), and vascular disease-1482 patients (36.8\%). Nonpermanent AF was present in 2549 patients (63.3\%).

A high risk of thromboembolic complications according to the $\mathrm{CHA}_{2} \mathrm{DS}_{2}$-VASc was reported in 3630 patients (90.1\%), and a high risk of bleeding complications according to the HAS-BLED was reported in 1179 patients $(29.3 \%)$. Table 1 presents the clinical characteristics in the study cohort.

3.2. Prevention of Thromboembolic Complications. In the study group of 4027 patients with AF, OACs were used in 3680 patients $(91.4 \%)$, antiplatelet drug/drugs in 124 patients (3.1\%), and 223 patients (5.5\%) did not undergo any thromboembolic event prevention.

In the group of patients treated with OACs, 1369 of them (37.2\%) were administered VKAs and 2311 (62.8\%) NOACs. In the group receiving NOACs, dabigatran was used in $51.4 \%$ of patients, rivaroxaban in $34.6 \%$, and apixaban in $14 \%$ of them.

Between 2014 and 2018, the increase in the proportion of NOAC-treated patients was observed from $30.5 \%$ to $70.1 \%$ (Figure 1). Figure 2 presents the proportion of patients treated with OACs in particular years of hospitalization.

Figure 3 shows the proportion of patients treated with individual OACs in particular categories according to the $\mathrm{CHA}_{2} \mathrm{DS}_{2}$-VASc score.

3.3. Comparison of Patients Treated with VKAs and NOACs. In the group of 3680 patients treated with OACs, 2311 patients $(62.8 \%)$ received NOACs and 1639 of them $(37.2 \%)$ were administered VKAs. NOAC patients in comparison to VKA patients were older [72.2(11.5) vs. 71.3(10); $P=0.001]$ and more often diagnosed with past thromboembolic complications $(13.9 \%$ vs. $11.6 \% ; P=0.047)$ and nonpermanent AF $(68.8 \%$ vs. $52 \%$; $P<0.001)$. The $\mathrm{CHA}_{2} \mathrm{DS}_{2^{-}}$ VASc score was similar in patients treated with VKAs and NOACs [3.9(1.9) vs. 4(1.8); $P=0.34$ ] whereas the HASBLED score was higher in VKA patients compared with 
TABLE 1: Baseline characteristics of the study group.

\begin{tabular}{|c|c|c|c|c|}
\hline Variable & All $n=4027$ & OAC $n=3680$ & APT $n=124$ & None $n=223$ \\
\hline Female, $n(\%)$ & $1714(42.6)$ & $1572(42.7)$ & $45(36.3)$ & $97(43.5)$ \\
\hline Age, mean (SD), years & $71.7(11.4)$ & $71.8(12)$ & $71.4(13.4)$ & $70.2(15.5)$ \\
\hline \multicolumn{5}{|l|}{ Clinical characteristics, $n(\%)$} \\
\hline Heart failure & $2520(62.6)$ & $2276(61.8)$ & $85(68.5)$ & $159(71.3)$ \\
\hline Hypertension & $3117(77.4)$ & $2891(78.6)$ & $79(63.7)$ & $147(65.9)$ \\
\hline Vascular disease & $1482(36.8)$ & $1331(36.2)$ & $79(63.7)$ & $72(32.3)$ \\
\hline Diabetes mellitus & $1148(28.5)$ & $1063(28.9)$ & $30(24.2)$ & $55(24.7)$ \\
\hline Previous thromboembolic event & $514(12.8)$ & $480(13.0)$ & $14(11.3)$ & $20(9)$ \\
\hline \multicolumn{5}{|l|}{ AF type, $n(\%)$} \\
\hline Paroxysmal & $1788(44.4)$ & $1566(42.6)$ & $99(79.8)$ & $123(55.2)$ \\
\hline Persistent & $761(18.9)$ & $737(20.0)$ & $5(4.1)$ & $19(8.5)$ \\
\hline Permanent & $1478(36.7)$ & $1377(37.4)$ & $20(16.1)$ & $81(36.3)$ \\
\hline Nonpermanent & $2549(63.3)$ & $2303(62.6)$ & $104(83.9)$ & $142(63.7)$ \\
\hline \multicolumn{5}{|l|}{ Thromboembolic risk } \\
\hline CHA2DS2-VASc mean (SD) & $3.9(1.8)$ & $3.9(1.8)$ & $4.0(1.8)$ & $3.7(2.0)$ \\
\hline $\mathrm{CHA} 2 \mathrm{DS} 2-\mathrm{VASc}=0, n(\%)$ & $99(2.5)$ & $74(2.0)$ & $4(3.2)$ & $21(9.4)$ \\
\hline $\mathrm{CHA} 2 \mathrm{DS} 2-\mathrm{VASc}=1, n(\%)$ & $298(7.4)$ & $271(7.4)$ & $7(5.6)$ & $20(9.0)$ \\
\hline $\mathrm{CHA} 2 \mathrm{DS} 2-\mathrm{VASc} \geq 2, n(\%)$ & $3630(90.1)$ & $3335(90.6)$ & $113(91.2)$ & $182(81.6)$ \\
\hline \multicolumn{5}{|l|}{ Bleeding risk } \\
\hline HAS-BLED, mean (SD) & $2.0(1.0)$ & $2.0(0.9)$ & $2.0(1.1)$ & $2.0(1.1)$ \\
\hline HAS-BLED $\geq 3, n(\%)$ & $1179(29.3)$ & $1064(28.9)$ & $36(29)$ & $79(35.4)$ \\
\hline \multicolumn{5}{|l|}{ Laboratory tests } \\
\hline $\mathrm{HGB}, \mathrm{g} / \mathrm{dl}$ & $n=3957$ & $n=3615$ & $n=124$ & $n=218$ \\
\hline Mean (SD) & $13.3(1.8)$ & $13.4(1.7)$ & $12.8(2.3)$ & $12.8(2.5)$ \\
\hline PLT, 103/ul & $n=3930$ & $n=3594$ & $n=121$ & $n=215$ \\
\hline Mean (SD) & $212.0(75)$ & $210.6(72.8)$ & $235.1(88.2)$ & $223.2(95.7)$ \\
\hline Creatinine, $\mathrm{mg} / \mathrm{dl}$ & $n=4008$ & $n=3668$ & $n=123$ & $n=217$ \\
\hline Mean (SD) & $1.3(0.5)$ & $1.3(0.4)$ & $1.2(0.4)$ & $1.4(0.8)$ \\
\hline $\mathrm{eGFR}, 60 \mathrm{ml} / \mathrm{min} / 1.73 \mathrm{~m}^{2}$ & $n=4008$ & $n=3679$ & $n=123$ & $n=217$ \\
\hline Mean (SD) & $54.9(16.5)$ & $54.8(16.3)$ & $57.9(16.4)$ & $53.6(20)$ \\
\hline $\mathrm{eGFR}<60 \mathrm{ml} / \mathrm{min} / 1.73 \mathrm{~m}^{2}, n(\%)$ & $2558(63.8)$ & $2355(64.0)$ & $71(57.7)$ & $68(31.3)$ \\
\hline \multicolumn{5}{|l|}{ Echocardiography } \\
\hline $\mathrm{LA}, \mathrm{mm}$ & $n=2944$ & $n=2667$ & $n=106$ & $n=171$ \\
\hline Mean (SD) & $46.9(7.8)$ & $47.1(7.7)$ & $43.8(7.3)$ & $44.7(8.9)$ \\
\hline LVDD, mm & $n=2960$ & $n=2680$ & $n=107$ & $n=173$ \\
\hline Mean (SD) & $52.9(8.8)$ & $53.1(8.7)$ & $51.8(9.1)$ & $50.7(9.8)$ \\
\hline LVEF, (\%) & $n=2985$ & $n=2704$ & $n=108$ & $n=173$ \\
\hline Mean (SD) & $48.3(16.7)$ & $48.2(17.1)$ & $47.3(11.6)$ & $49.7(14.3)$ \\
\hline
\end{tabular}

Data are presented as number (percentage) or mean (standard deviation) (SD) or median (interquartile range) (IQR). AF: atrial fibrillation; APT: antiplatelet drug/drugs; eGFR: estimated glomerular filtration rate; LA: left atrium; LVDD: left ventricular end-diastolic dimension; LVEF: left ventricular ejection fraction; and OAC: oral anticoagulant.

NOAC ones [2(1) vs. 2(0.9); $P=0.005$ ]. The eGFR $<60 \mathrm{ml} /$ $\mathrm{min} / 1.73 \mathrm{~m} 2$ statistically significantly more often occurred in the group of VKA patients than in the NOAC one $(67.1 \%$ vs. $62.4 \% ; P=0.008)$. Patients treated with VKAs more often than NOAC patients were administered antiplatelet pharmaceutical/pharmaceuticals $(9.7 \%$ vs. $6.1 \% ; \quad P<0.001)$ (Table 2).

3.4. Predictors of NOAC Choice in the Group of Patients Receiving $O A C s$. In the univariable logistic regression analysis, numerous predictors of NOAC prescription were found (Table S1). In the multivariable model, factors associated with the selection of NOACs versus VKAs included the following: age, eGFR, a previous thromboembolic event, nonpermanent AF, left atrial (LA) diameter and antiplatelet drug/drugs.
Table 3 shows the predictors of the NOAC use-age (OR, 1.02 ; 95\% CI, 1.01-1.03; $P<0.001$ ), a previous thromboembolic event (OR, 1.29; 95\% CI, 1.01-1.65; $P=0.04$ ), nonpermanent AF (OR, 1.61; 95\% CI, 1.34-1.93; $P<0.001)$, and eGFR (OR, 1.22; 95\% CI, 1.02-1.46; $P=0.03$ ).

LA diameter (OR, 0.97; CI, 0.96-0.98; $P<0.001)$ and antiplatelet drug/drugs (OR, 0.68; CI, 0.5-10.92; $P=0.01$ ) diminished the chance of choosing NOACs.

\section{Discussion}

Many studies have reported general improvement of $\mathrm{AF}$ patient management in terms of OAC treatment after NOACs were introduced $[14,15]$. The same applies to the steady decrease in VKA and antiplatelet drug use in favour of NOAC therapies. Similar results were achieved in the 


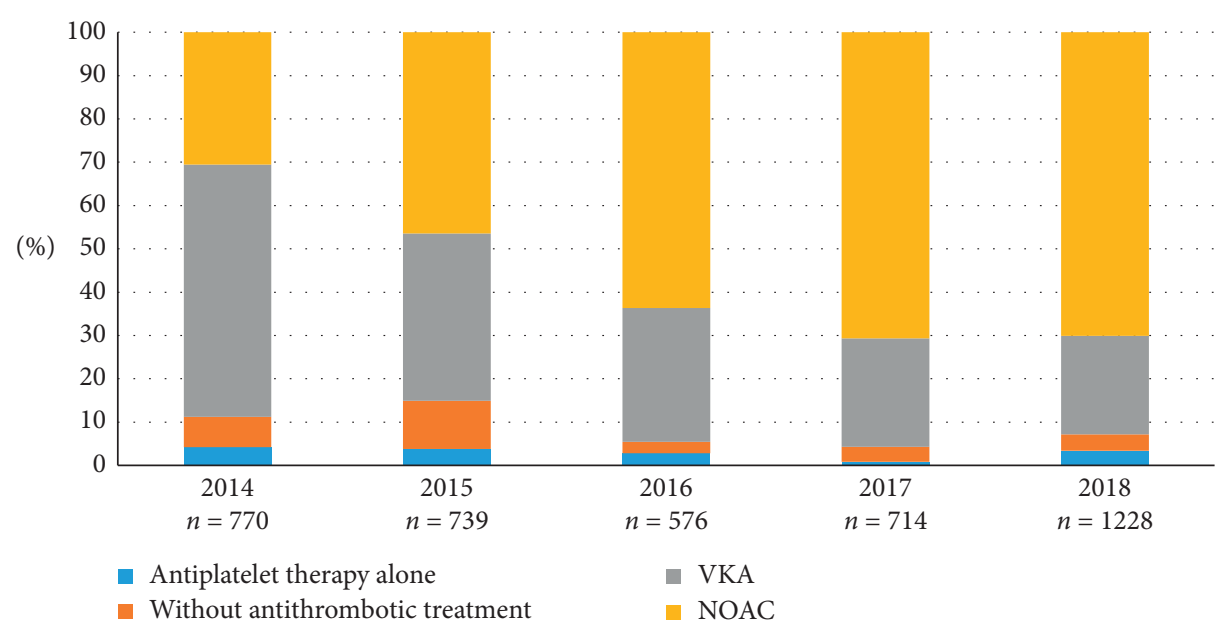

FIGURE 1: Stroke prophylaxis in patients with atrial fibrillation in particular years of hospitalization.

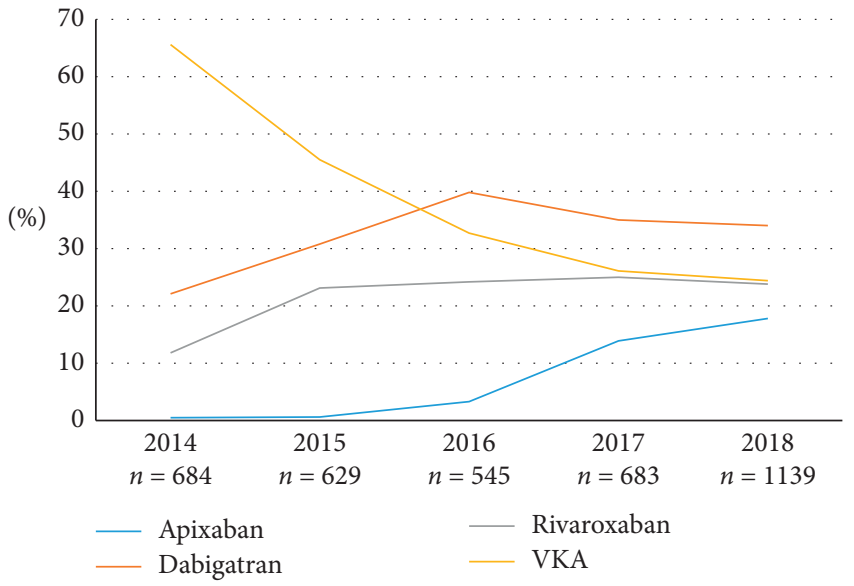

FIGURE 2: Proportion of patients treated with oral anticoagulants in particular years of hospitalization.

present study, which in particular demonstrates new data concerning prescription of OACs as well as NOACs and VKAs in AF patients from Poland.

In recent years, using OACs in AF treatment gradually increased worldwide ( $>80 \%)$. However, regional differences are clearly visible-the highest uptake in Europe (90\%) and North America (78.2\%) and the lowest one in Asia (57.4\%). Interestingly, the percentage of patients receiving only antiplatelet drugs or untreated decreased, even if it was still high in the patients of high risk [16]. In the presented group of 4027 patients with AF, $91 \%$ of them received OACs. In the All Nippon AF in the Elderly (ANAFIE) Registry, 92\% of 32726 patients aged $\geq 75$ years were administered OACs [17]. Similarly, the Atrial Fibrillation in Octogenarians (OCTOFA) Study showed that $92 \%$ of 738 patients $\geq 80$ years received OACs [18]. In the EURObservational Research Programme on Atrial Fibrillation (EORP-AF) LongTerm General Registry, OACs were used in $84.9 \%$ of patients [19].

In the present study, NOACs were used in $63 \%$ of patients treated with OACs, and a significant increase in the proportion of patients treated with NOACs was observed over five years. In 2014, they constituted $34.4 \%$ of OACs and in 2018 it was 75.6\%. Many studies clearly outline a significant implementation of NOACs in thromboembolic prophylaxis of AF in recent years. In Global Anticoagulant Registry in the FIELD-Atrial Fibrillation (GARFIELD-AF), the proportion of prescribed NOACs rose from $34 \%$ to $62 \%$ within 3 years [20]. EORP-AF General Long-Term General Registry in comparison to EORP-AF Pilot showed that during four years there was a NOAC prescription increase from less than $10 \%$ of patients to about $35 \%[19,21]$. From 2011 to 2016, Balsam et al. observed an increase of prescribed NOACs to $2 / 3$ of all OACs [22].

In the present study, dabigatran was the most frequently prescribed NOAC. Dabigatran was the first, rivaroxaban the second, and apixaban the third NOAC available in Poland, and all the drugs were available in the whole study period. Our study showed a strong increase in apixaban use since its introduction. It was higher than the increase in dabigatran and rivaroxaban use.

In the present study, antiplatelet drug was received by $3.1 \%$ of patients (124 out of 4,027), and 5.5\% of patients were discharged from a hospital without any kind of anticoagulant therapy. Within 5 years, the proportion of patients with AF treated with antiplatelet pharmaceuticals decreased from $4.2 \%$ to $3.4 \%$, and patients without anticoagulant prophylaxis decreased from $7 \%$ to $3.8 \%$. According to the BLITZ-AF study, $9.1 \%$ of patients received only antiplatelet drugs and $7.5 \%$ were not treated with any antithrombotic drugs [23]. There are similar results from the Global Registry on Long-Term Oral Antithrombotic Treatment in Patients with Atrial Fibrillation Registry (GLORIA-AF) where $12.1 \%$ were on antiplatelets and $7.8 \%$ were not on antithrombotic therapy [24]. In light of the effectual guidelines related to AF, antiplatelet pharmaceuticals should not be used in the prevention of thromboembolic complications in patients with AF [4]. However, it happens that they are used against the guidelines in situations where OAC administration seems to be too risky.

In our study, we identified independent predictors of NOAC use and it is a novel finding of specific interest. In the present study, the predisposing factors for the use of NOACs 


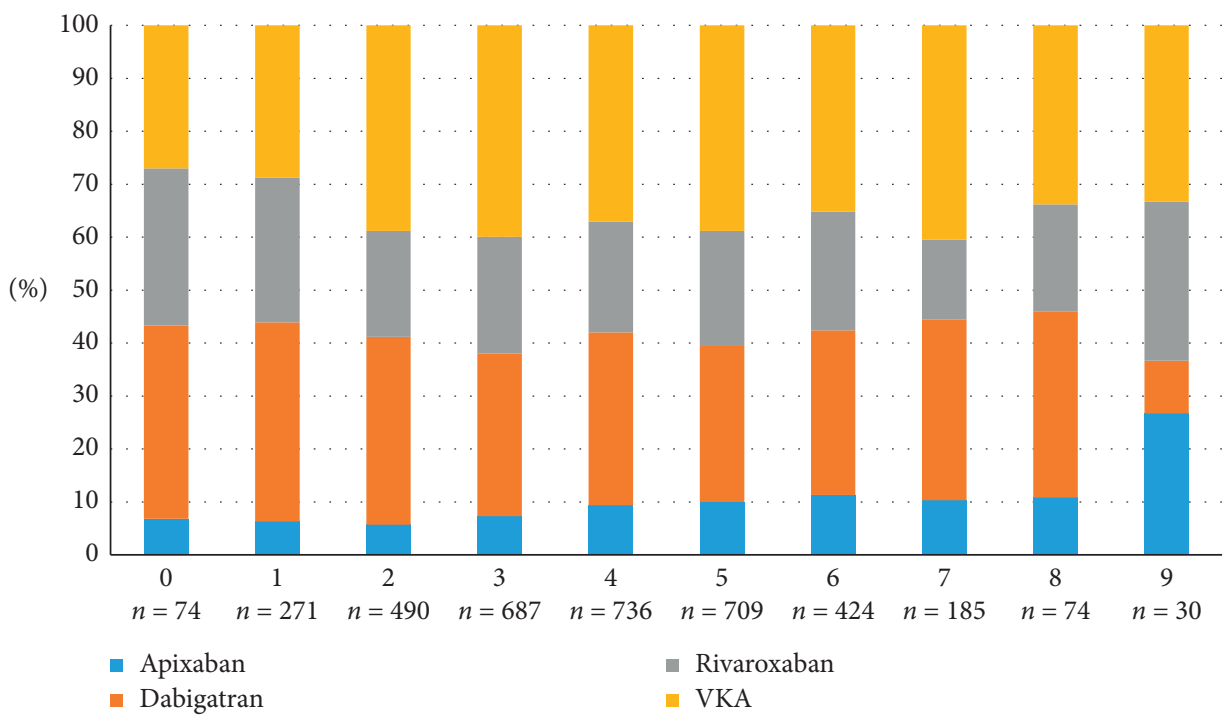

Figure 3: Prescription pattern of oral anticoagulants based on CHA2DS2-VASc score.

among AF patients were age, eGFR, a previous thromboembolic event, nonpermanent AF, while the predisposing factors for VKA use were LA diameter and antiplatelet drug/drugs. Although the current guidelines make no distinction between nonpermanent and permanent $\mathrm{AF}$ for stroke prevention, clinical data confirmed that the type of $\mathrm{AF}$ was associated with an increased risk of stroke [25]. It is possible then that, in the future, the AF type will be included in a thromboembolic risk stratification and choosing anticoagulant prophylaxis. In the present study, the strongest predictor of NOAC vs. VKA choice was nonpermanent AF. Similarly, in the group of patients in GARFIELD-AF Registry, NOACs were preferred in patients with paroxysmal AF [26]. Choosing NOACs in patients with nonpermanent $\mathrm{AF}$ is connected with the fact that they are usually de novo AF patients and when there is a decision to use OACs, according to the guidelines, NOACs should be preferred [4].

NOACs are preferred after a stroke and other thromboembolic events [4]. In the present study, a previous thromboembolic event was a strong predictor for the choice of NOAC. Thromboembolic events were observed in $13 \%$ of patients with AF. A similar proportion of patients with thromboembolic complications was noticed in PREvention of thromboembolic events-European Registry in Atrial Fibrillation (PREFER) Registry-8.4\% of patients [27]. Gorczyca et al. [28] showed that in AF patients after a thromboembolic event, $59 \%$ of patients on OACs were treated with NOACs. The Novel Oral Anticoagulants in Stroke Patients (NOACISP LONG-TERM) Registry showed that NOACs were used in $78 \%$ of patients treated with OACs in the secondary prevention of thromboembolic events [29].

Impaired renal function is an acknowledged risk factor of thrombus formation, stroke, systemic embolism, and bleeding events. Data showed that NOACs would reduce the risk of stroke or systemic embolism and major bleeding concerning different levels of renal function [30-32]. In the present study, it was shown that higher eGFR predisposes the choice of NOACs. NOACs are contraindicated in patients with significant impairment of renal function.

Elderly patients more often choose NOACs in the study group which complies with the GARFIELD Registry results [26] and the ANAFIE study [17]. In older patients with AF, NOACs have a better efficacy and safety profile than VKAs [33]. In contrast, the results from the Outcomes Registry for Better Informed Treatment of Atrial Fibrillation II (ORBIT$\mathrm{AF}$ II) showed that patients treated with NOACs were younger than patients treated with VKAs [34]. It is difficult to compare the data obtained from different registries because their results are dependent on the clinical characteristics of patients and the preferences of doctors. The data depends also on the geographical region.

In the present study, LA extension was a predictor of VKA usage. It is possible that patients with extended LA had longer-lasting $\mathrm{AF}$ than patients with the normal LA diameter and in these patients, VKAs have been administered for many years.

A factor predisposing the choice of VKA in the prevention of thromboembolic complications was also the application of antiplatelet drug/drugs. Balsam et al. [22] showed that VKA patients were more often treated with antiplatelet pharmaceuticals than patients receiving NOACs. Similarly, in the GARFIELD Registry, use of antiplatelet medications was a predictor of choosing VKAs; however, interestingly, acute coronary syndrome clinched the choice of NOAC [26]. It seems that, in clinical practice, adjusting the NOAC dose to a clinical risk and benefit balance may cause some problems when using NOACs and antiplatelet drugs concomitantly. The current guidelines regarding chronic coronary syndromes allow prescribing the above in a full or reduced dose when using dabigatran and rivaroxaban, depending on the evaluation of individual haemorrhagic and ischemic risks [35]. In the present study, cancer was not a predictor of anticoagulant choice. Mariani et al. [36] showed that NOACs, in comparison to VKA, were associated with a significant reduction of the rates of 
TABLE 2: Clinical characteristics of patients with atrial fibrillation treated with vitamin $\mathrm{K}$ antagonist and non-vitamin $\mathrm{K}$ antagonist oral anticoagulant.

\begin{tabular}{|c|c|c|c|c|}
\hline Clinical feature & OAC $n=3680$ & NOAC $n=2311$ & VKA $n=1369$ & $P$ \\
\hline Female, $n(\%)$ & $1572(42.7)$ & $1008(43.6)$ & $564(41.2)$ & 0.152 \\
\hline \multicolumn{5}{|l|}{ Age, years } \\
\hline Mean (SD) & $71.8(12)$ & $72.2(11.5)$ & $71.3(10)$ & 0.001 \\
\hline Median (IQR) & $72(15)$ & $73(16)$ & $71(14)$ & \\
\hline \multicolumn{5}{|l|}{ Clinical characteristics, $n$ (\%) } \\
\hline Heart failure & $2276(61.8)$ & $1338(57.9)$ & $938(68.5)$ & $<0.001$ \\
\hline Hypertension & $2891(78.6)$ & $1804(78.1)$ & $1087(79.4)$ & 0.339 \\
\hline Diabetes mellitus & $1063(28.9)$ & $618(26.7)$ & $445(32.5)$ & $<0.001$ \\
\hline Previous thromboembolic event & $480(13)$ & $321(13.9)$ & $159(11.6)$ & 0.047 \\
\hline Vascular disease & $1331(36.2)$ & $800(34.6)$ & $531(38.8)$ & 0.011 \\
\hline Cancer & $157(4.3)$ & $102(4.4)$ & $55(4.0)$ & 0.345 \\
\hline \multicolumn{5}{|l|}{ AF type, $n(\%)$} \\
\hline Paroxysmal & $1566(42.6)$ & $1067(46.2)$ & $499(36.4)$ & $<0.001$ \\
\hline Persistent & $737(20.0)$ & $524(22.7)$ & $213(15.6)$ & $<0.001$ \\
\hline Permanent & $1377(37.4)$ & $720(31.2)$ & $657(48.0)$ & $<0.001$ \\
\hline Nonpermanent & $2303(62.6)$ & $1591(68.8)$ & $712(52.0)$ & $<0.001$ \\
\hline \multicolumn{5}{|l|}{ Thromboembolic risk } \\
\hline \multicolumn{5}{|l|}{ CHA2DS2-VASC score } \\
\hline Mean (SD) & $3.9(1.8)$ & $3.9(1.9)$ & $4.0(1.8)$ & 0.338 \\
\hline Median (IQR) & $4.0(2.0)$ & $4.0(2.0)$ & $4.0(2.0)$ & \\
\hline $\mathrm{CHA}_{2} \mathrm{DS}_{2}-\mathrm{VASC}=0, n(\%)$ & $74(2)$ & $54(2.3)$ & $20(1.5)$ & \\
\hline $\mathrm{CHA}_{2} \mathrm{DS}_{2}-\mathrm{VASC}=1, n(\%)$ & $271(7.4)$ & $193(8.4)$ & $78(5.7)$ & 0.002 \\
\hline $\mathrm{CHA} 2 \mathrm{DS} 2-\mathrm{VASC} \geq 2, n(\%)$ & $3335(90.6)$ & $2064(89.3)$ & $1271(92.8)$ & \\
\hline \multicolumn{5}{|l|}{ Bleeding risk } \\
\hline \multicolumn{5}{|l|}{ HASBLED score } \\
\hline Mean (SD) & $2.0(0.9)$ & $2.0(0.9)$ & $2.0(1.0)$ & 0.005 \\
\hline Median (IQR) & $2.0(2.0)$ & $2.0(2.0)$ & $2.0(2.0)$ & \\
\hline HASBLED $\geq 3, n(\%)$ & $1064(28.9)$ & $621(26.8)$ & $443(32.4)$ & 0.048 \\
\hline \multicolumn{5}{|l|}{ Laboratory tests } \\
\hline$H G B, g / d l$ & $n=3615$ & $n=2268$ & $n=1347$ & \\
\hline Mean (SD) & $13.4(1.7)$ & $13.4(1.7)$ & $13.3(1.7)$ & 0.091 \\
\hline Median (IQR) & $13.5(2.2)$ & $13.5(2.3)$ & $13.4(2.1)$ & \\
\hline$P L T, 103 / u l$ & $n=3594$ & $n=2255$ & $n=1339$ & \\
\hline Mean (SD) & $210.6(72.8)$ & $215.3(75.5)$ & $202.6(67.1)$ & $<0.001$ \\
\hline Median (IQR) & $200.0(78.0)$ & $205.0(78.0)$ & $193.0(74.0)$ & \\
\hline Creatinine, $\mathrm{mg} / \mathrm{dl}$ & $n=3668$ & $n=2304$ & $n=1364$ & \\
\hline Mean (SD) & $1.3(0.4)$ & $1.2(0.3)$ & $1.3(0.5)$ & $<0.001$ \\
\hline Median (IQR) & $1.2(0.4)$ & $1.2(0.3)$ & $1.2(0.4)$ & \\
\hline$e G F R, 60 \mathrm{ml} / \mathrm{min} / 1.73 \mathrm{~m}^{2}$ & $n=3679$ & $n=2311$ & $n=1368$ & \\
\hline Mean (SD) & $54.8(16.3)$ & $55.8(16.1)$ & $53.3(16.4)$ & $<0.001$ \\
\hline Median (IQR) & $54.2(21.1)$ & $55.1(21.3)$ & $52.7(20.7)$ & \\
\hline $\mathrm{eGFR}<60 \mathrm{ml} / \mathrm{min} / 1.73 \mathrm{~m}^{2}, n(\%)$ & $2355(64.0)$ & $1442(62.4)$ & $913(67.1)$ & 0.008 \\
\hline \multicolumn{5}{|l|}{ Echocardiography } \\
\hline$L A, \mathrm{~mm}$ & $n=2667$ & $n=1664$ & $n=1003$ & \\
\hline Mean (SD) & $47.1(7.7)$ & $46.1(7.3)$ & $48.8(8.1)$ & $<0.001$ \\
\hline Median (IQR) & $46.0(10.0)$ & $45.0(9.0)$ & $48.0(10.0)$ & \\
\hline$L V D D, m m$ & $n=2680$ & $n=1676$ & $n=1004$ & \\
\hline Mean (SD) & $53.1(8.7)$ & $52.3(8.4)$ & $54.4(9.0)$ & $<0.001$ \\
\hline Median (IQR) & $52.0(11.0)$ & $51.0(10.0)$ & $53.0(11.0)$ & \\
\hline$L V E F, n(\%)$ & $n=2704$ & $n=1688$ & $n=1016$ & \\
\hline Mean (SD) & $48.2(17.1)$ & $49.5(18.8)$ & $46.1(13.7)$ & $<0.001$ \\
\hline Median (IQR) & $50.0(20.0)$ & $52.0(18.0)$ & $50.0(19.0)$ & \\
\hline Antiplatelet drug/drugs, $n(\%)$ & $275(7.5)$ & $142(6.1)$ & $133(9.7)$ & $<0.001$ \\
\hline
\end{tabular}

Data are presented as number (percentage) or mean (standard deviation) (SD) or median (interquartile range) (IQR). AF: atrial fibrillation; eGFR: estimated glomerular filtration rate; HGB: hemoglobin concentration; LA: left atrium; LVDD: left ventricular end-diastolic dysfunction; LVEF: left ventricular ejection fraction; NOAC: non-vitamin K antagonist oral anticoagulant; OAC: oral anticoagulation therapy; PLT: platelet count; TIA: transient ischemic attack; and VKA: vitamin $\mathrm{K}$ antagonist oral anticoagulants. 
TABLE 3: Factors increasing the chances of using non-vitamin $\mathrm{K}$ antagonist oral anticoagulant in analysed patients-multivariate logistic regression analysis.

\begin{tabular}{lccc}
\hline Factors & OR & $95 \%$ CI & $P$ \\
\hline Age (per year) & 1.02 & $1.01-1.03$ & $<0.001$ \\
Heart failure & 0.84 & $0.70-1.00$ & 0.057 \\
Diabetes mellitus & 0.88 & $0.74-1.05$ & 0.163 \\
Previous thromboembolism event & 1.29 & $1.01-1.65$ & 0.038 \\
Vascular disease & 0.91 & $0.77-1.09$ & 0.320 \\
Nonpermanent AF & 1.61 & $1.34-1.93$ & $<0.001$ \\
eGFR (per unit) & 1.22 & $1.02-1.46$ & 0.030 \\
LA diameter & 0.97 & $0.96-0.98$ & $<0.001$ \\
Antiplatelet drug/drugs & 0.68 & $0.51-0.92$ & 0.011 \\
\hline
\end{tabular}

AF: atrial fibrillation; CI: confidence interval; eGFR: estimated glomerular filtration rate; LA: left atrium; and OR: odds ratio.

thromboembolic events and major bleeding complications in AF patients with cancer.

\section{Limitations}

This study has several limitations. Firstly, it was not possible to assess anticoagulation effects on outcomes due to collected data observational nature and no follow-up data. Secondly, the present study is a single-center registry, but it was conducted in the referential center, where ambulatory patients were sent, and in other hospitals. Thirdly, in the present study, most patients with $\mathrm{AF}$ were not OAC-naïve. Thus, despite the unicentric character of the study, it shows the anticoagulant therapy trends of doctors from particular regions referring patients to the center where the study comes from.

\section{Conclusions}

In the present "real-world" study, significant changes in the prophylaxis of thromboembolic complications in patients with AF within recent years were reported. The number of patients treated with OACs increased significantly. In most of them, NOACs were administered. Age, previous thromboembolic complications, nonpermanent AF, and preserved renal function determined the choice of NOACs in patients treated with OACs.

\section{Data Availability}

The source data used to support the findings of this study are available from the corresponding author upon request.

\section{Conflicts of Interest}

Bernadetta Bielecka and Olga Jelonek have no conflicts of interest that might influence this work. Iwona Gorczyca received payment for lectures from Bayer and Boehringer Ingelheim. Beata Wożakowska-Kapłon received payment for lectures from Bayer, Boehringer Ingelheim, and Pfizer.

\section{Acknowledgments}

This work was supported by the Ministry of Science and Higher Education entitled "Regional Initiative of Excellence" in the years 2019-2022 (Project no. 024/RID/2018/19; amount of financing PLN 11999000).

\section{Supplementary Materials}

Table S1: factors increasing the chances of using nonvitamin $\mathrm{K}$ antagonist oral anticoagulants in analysed patients-univariable logistic regression analysis. (Supplementary Materials)

\section{References}

[1] P. A. Wolf, R. D. Abbott, and W. B. Kannel, "Atrial fibrillation as an independent risk factor for stroke: the Framingham study," Stroke, vol. 22, no. 8, pp. 983-988, 1991.

[2] A. S. Go, E. M. Hylek, K. A. Phillips et al., "Prevalence of diagnosed atrial fibrillation in adults," JAMA, vol. 285 , no. 18 , pp. 2370-2375, 2001.

[3] A. J. Camm, P. Kirchhof, A. J. Camm et al., "Guidelines for the management of atrial fibrillation: the task force for the management of atrial fibrillation of the European society of cardiology (ESC)," European Heart Journal, vol. 31, no. 19, pp. 2369-2429, 2010.

[4] P. Kirchhof, S. Benussi, and D. Kotecha, "ESC Guidelines for the management of atrial fibrillation developed in collaboration with EACTS," Europace, vol. 18, no. 11, pp. 1609-1678, 2016.

[5] R. G. Hart, L. A. Pearce, and M. I. Aguilar, "Meta-analysis: antithrombotic therapy to prevent stroke in patients who have nonvalvular atrial fibrillation," Annals of Internal Medicine, vol. 146, no. 12, pp. 857-867, 2007.

[6] C. B. Granger, J. H. Alexander, J. J. V. McMurray et al., "Apixaban versus warfarin in patients with atrial fibrillation," New England Journal of Medicine, vol. 365, no. 11, pp. 981992, 2011.

[7] S. J. Connolly, M. D. Ezekowitz, S. Yusuf et al., "Dabigatran versus warfarin in patients with atrial fibrillation," New England Journal of Medicine, vol. 361, no. 12, pp. 1139-1151, 2009.

[8] M. R. Patel, K. W. Mahaffey, J. Garg et al., "Rivaroxaban versus warfarin in nonvalvular atrial fibrillation," New England Journal of Medicine, vol. 365, no. 10, pp. 883-891, 2011.

[9] S. Perreault, S. Denus, B. White-Guay et al., "Oral anticoagulant prescription trends, profile use, and determinants of adherence in patients with atrial fibrillation," Pharmacotherapy: The Journal of Human Pharmacology and Drug Therapy, vol. 40, no. 1, pp. 40-54, 2020.

[10] M. E. Johnson, C. Lefèvre, S.-L. Collings et al., "Early realworld evidence of persistence on oral anticoagulants for stroke prevention in non-valvular atrial fibrillation: a cohort study in UK primary care," BMJ Open, vol. 6, no. 9, Article ID e011471, 2016.

[11] M. R. Fay, J. L. Martins, and B. Czekay, "Oral anticoagulant prescribing patterns for stroke prevention in atrial fibrillation among general practitioners and cardiologists in three European countries," European Heart Journal, vol. 37, p. 510, 2016.

[12] J. B. Olesen, R. Sorensen, M. L. Hansen et al., "Non-vitamin K antagonist oral anticoagulation agents in anticoagulant naive atrial fibrillation patients: Danish nationwide descriptive data 2011-2013," Europace, vol. 17, no. 2, pp. 187-193, 2015.

[13] L. Huiart, C. Ferdynus, and C. Renoux, "Trends in initiation of direct oral anticoagulant therapies for atrial fibrillation in a 
national population- based cross-sectional study in the French health insurance databases," BMJ Open, vol. 8, no. 3, 2018.

[14] P. Lopatowska, A. Tomaszuk-Kazberuk, E. Mlodawska et al., "Do CHA2 DS2 VASc and HAS-BLED scores influence 'realworld' anticoagulation management in atrial fibrillation? 1556 patient registry from the reference cardiology centre," Pharmacoepidemiology and Drug Safety, vol. 24, no. 12, pp. 1297-1303, 2015.

[15] G. Maura, C. Billionnet, and J. Drouin, "Oral anticoagulation therapy use in patients with atrial fibrillation after the introduction of nonvitamin $\mathrm{K}$ antagonist oral anticoagulants: findings from the French healthcare databases, 2011-2016," BMJ Open, vol. 9, no. 4, Article ID e026645, 2019.

[16] M. Mazurek, E. Shantsila, D. A. Lane, A. Wolff, M. Proietti, and G. Y. H. Lip, "Guideline-adherent antithrombotic treatment improves outcomes in patients with atrial fibrillation," Mayo Clinic Proceedings, vol. 92, no. 8, pp. 1203-1213, 2017.

[17] Y. Koretsune, T. Yamashita, M. Akao et al., "Baseline demographics and clinical characteristics in the all nippon AF in the elderly (ANAFIE) registry," Circulation Journal, vol. 83, no. 7, pp. 1538-1545, 2019.

[18] J. Blacher, E. Sorbets, D. Guedj Meynier et al., "Determinants of antithrombotic treatment for atrial fibrillation in octogenarians: results of the OCTOFA study," Clinical Drug Investigation, vol. 39, no. 9, pp. 891-898, 2019.

[19] G. Boriani, M. Proietti, C. Laroche et al., "Contemporary stroke prevention strategies in 11096 European patients with atrial fibrillation: a report from the EURObservational Research Programme on atrial fibrillation (EORP-AF) long-term general registry," Europace: European Pacing, Arrhythmias, and Cardiac Electrophysiology: Journal of the Working Groups on Cardiac Pacing, Arrhythmias, and Cardiac Cellular Electrophysiology of the European Society of Cardiology, vol. 20, no. 5, pp. 747-757, 2018.

[20] J. P. Bassand, G. Accetta, and W. Al Mahmeed, "Risk factors for death, stroke, and bleeding in 28,628 patients from the GARFIELD-AF registry: rationale for comprehensive management of atrial fibrillation," PLoS One, vol. 13, no. 1, Article ID e0191592, 2018.

[21] G. Y. H. Lip, C. Laroche, G.-A. Dan et al., "A prospective survey in European Society of Cardiology member countries of atrial fibrillation management: baseline results of EURObservational Research Programme Atrial Fibrillation (EORPAF) Pilot General Registry," EP Europace, vol. 16, no. 3, pp. 308-319, 2014.

[22] P. Balsam, K. Ozierański, A. Tymińska et al., "Comparison of clinical characteristics of real-life atrial fibrillation patients treated with vitamin $\mathrm{K}$ antagonists, dabigatran, and rivaroxaban: results from the CRAFT study," Kardiologia Polska, vol. 76, no. 5, pp. 889-898, 2018.

[23] R. Cemin, F. Colivicchi, A. P. Maggioni et al., "One-year clinical events and management of patients with atrial fibrillation hospitalized in cardiology centers: data from the BLITZ-AF study," European Journal of Internal Medicine, vol. 74, pp. 55-60, 2020.

[24] M. V. Huisman, K. J. Rothmann, and M. Paquette, "The changing landscape for stroke prevention in AF. Findings from the GLORIA-AF registry phase 2," Journal of the American College of Cardiology, vol. 69, no. 7, pp. 777-785, 2017.

[25] S. Cho, J. Kim, J.-B. Kim et al., "The difference of burden of ectopic beats in different types of atrial fibrillation and the effect of atrial fibrillation type on stroke risk in a prospective cohort of patients with atrial fibrillation (CODE-AF registry)," Scientific Reports, vol. 10, no. 1, 2020.

[26] S. Haas, A. J. Camm, J.-P. Bassand et al., "Predictors of NOAC versus VKA use for stroke prevention in patients with newly diagnosed atrial fibrillation: results from GARFIELD-AF," American Heart Journal, vol. 213, pp. 35-46, 2019.

[27] P. Kirchhof, B. Ammentorp, H. Darius et al., "Management of atrial fibrillation in seven European countries after the publication of the 2010 ESC Guidelines on atrial fibrillation: primary results of the PREvention oF thromboemolic eventsEuropean Registry in Atrial Fibrillation (PREFER in AF)," EP Europace, vol. 16, no. 1, pp. 6-14, 2014.

[28] I. Gorczyca, A. Michalska, M. Chrapek, O. Jelonek, P. Wałek, and B. Wożakowska-Kapłon, "Non-vitamin K antagonist oral anticoagulants in patients with atrial fibrillation in secondary stroke and systemic embolism prevention," Cardiology Journal, 2019.

[29] D. J. Seiffge, C. Traenka, A. Polymeris et al., "Early start of DOAC after ischemic stroke," Neurology, vol. 87, no. 18, pp. 1856-1862, 2016.

[30] F. Del-Carpio Munoz, S. M. Gharacholou, T. M. Munger et al., "Meta-analysis of renal function on the safety and efficacy of novel oral anticoagulants for atrial fibrillation," The American Journal of Cardiology, vol. 117, no. 1, pp. 69-75, 2016.

[31] R. Zou, J. Tao, W. Shi et al., "Meta-analysis of safety and efficacy for direct oral anticoagulation treatment of nonvalvular atrial fibrillation in relation to renal function," Thrombosis Research, vol. 160, pp. 41-50, 2017.

[32] A. Bociek, A. Bielejewska, M. Bociek, T. Dereziński, and A. Jaroszyński, "The bidirectional relationship between chronic kidney disease and atrial fibrillation does not occur in elderly female outpatients," Medical Studies, vol. 35, no. 3, pp. 185-189, 2019.

[33] I.-S. Kim, H.-J. Kim, T.-H. Kim et al., "Non-vitamin K antagonist oral anticoagulants have better efficacy and equivalent safety compared to warfarin in elderly patients with atrial fibrillation: a systematic review and meta-analysis," Journal of Cardiology, vol. 72, no. 2, pp. 105-112, 2018.

[34] B. A. Steinberg, P. Shrader, L. Thomas et al., "Factors associated with non-vitamin $\mathrm{K}$ antagonist oral anticoagulants for stroke prevention in patients with new-onset atrial fibrillation: results from the Outcomes Registry for Better Informed Treatment of Atrial Fibrillation II (ORBIT-AF II)," American Heart Journal, vol. 189, pp. 40-47, 2017.

[35] J. Knuuti, W. Wijns, and A. Saraste, "Guidelines for the diagnosis and management of chronic coronary syndromes," European Heart Journal, vol. 41, no. 3, pp. 407-477, 2020.

[36] M. V. Mariani, M. Magnocavallo, M. Straito et al., "Direct oral anticoagulants versus vitamin $\mathrm{K}$ antagonists in patients with atrial fibrillation and cancer a meta-analysis," Journal of Thrombosis and Thrombolysis, 2020. 